Revue d'histoire de l'Amérique française

ZRS REVUE D.HISTOIRE DE L'AMÉRIQUE FRANÇAISE

\title{
Un exemple de peinture d'histoire au Québec : Charles Huot à l'Assemblée nationale
}

\section{Raymond Montpetit}

Volume 31, numéro 3, décembre 1977

URI : https://id.erudit.org/iderudit/303635ar

DOI : https://doi.org/10.7202/303635ar

Aller au sommaire du numéro

Éditeur(s)

Institut d'histoire de l'Amérique française

ISSN

0035-2357 (imprimé)

1492-1383 (numérique)

Découvrir la revue

Citer cette note

Montpetit, R. (1977). Un exemple de peinture d'histoire au Québec : Charles Huot à l'Assemblée nationale. Revue d'histoire de l'Amérique française, 31(3), 397-405. https://doi.org/10.7202/303635ar d'utilisation que vous pouvez consulter en ligne. 
NOTES DE RECHERCHE

\title{
UN EXEMPLE DE PEINTURE D'HISTOIRE AU QUÉBEC: CHARLES HUOT À L'ASSEMBLÉE NATIONALE*
}

\author{
RAYMOND MONTPETIT \\ Département d'bistoire de l'art \\ Université du Québec à Montréal
}

Par l'entremise de certaines images peintes, les illettrés contemplent ce qu'ils ne pourraient saisir par l'écriture.

Synode d'Arras 1025

S'il est un terrain de prédilection pour situer l'articulation histoire de l'art/histoire, c'est bien celui de la peinture d'histoire; bien sûr, tant que les historiens de l'art restent subjugués par les dites «valeurs esthétiques» et par le mérite respectif des artistes qui en sont les dépositaires, et tant que les historiens se cantonnent dans l'analyse des seules sources écrites, la peinture d'histoire demeure hors de leurs champs d'analyse, puisque de telles productions ne sont pas toujours marquantes dans l'évolution des schèmes esthétiques, ni ne semblent constituer des documents qui puissent ajouter à la connaissance des événements, des perspectives nouvelles.

La publication de Robert Derome a, dès le départ, le mérite de sortir de l'ombre une pratique picturale, en l'occurrence celle de Charles Huot, qui s'inscrit de plein pied dans une tradition de peinture d'histoire, qui compte chez nous quantité d'œuvres.

* Récemment Robert Derome publiait, dans le Bulletin 27/1976 de la Galerie nationale du Canada, une étude intitulée "Charles Huot et la peinture d'histoire au Palais législatif de Québec (1883-1930)»; les réflexions qui suivent sont celles que nous avons énoncées en commentaire à la suite de la conférence qu'il a prononcée sur le même sujet, à l'occasion du dernier congrès de l'Institut d'histoire de l'Amérique française.

RHAF, vol. $31, \mathrm{n}^{\circ} 3$ (décembre 1977) 
Nous voudrions, à partir de son texte et de sa communication, mettre l'accent sur quelques pistes de réflexion autour des problèmes que pose une peinture d'histoire, une peinture qui se veut, après coup, la visibilité d'événements qu'une culture élit, et dans lesquels elle choisit explicitement de reconnaître son passé et, jusqu'à un certain point, son présent. C'est toujours à partir de ce présent que l'on se recommande du passé et qu'on commande au peintre une représentation qui le réactive et le mette en scène. Jamais autant que dans ce genre de peinture, le temps représenté (l'action dépeinte) et le temps de la représentation (l'année de l'exécution du tableau) ne se croisent et s'interpénètrent avec autant de pertinence et de conséquence quant à la signification même de l'œuvre.

La peinture d'histoire est, de par nature, un type d'expression dominé par le textuel; on peut presque la considérer comme étant une forme d'illustration, comme la représentation iconographique d'un récit textuel déterminé, d'un paragraphe d'histoire. En examinant les trois œuvres de Charles Huot qui ornent l'Assemblée nationaie du Québec, on se rend compte que toutes trois entretiennent avec l'événement et la littérature qui en est l'inscription, une relation différente:

1. «Le débat sur les langues: séance de l'Assemblée législative du Bas-Canada le 21 janvier 1793 », se source très ponctuellement dans un événement précis et daté, le débat qui marqua l'accession du Canada français au régime parlementaire et le choix d'une langue pour consigner les travaux du parlement.

2. «Le Conseil Souverain» est la représentation non pas d'une action déterminée que l'on pourrait citer, mais relève d'une temporalité floue, en fixant sur la toile plus la vision synthétique d'un régime que la description d'un fait connu ou nommable.

3. «Je me souviens» se veut une fresque, un panorama de temporalité abstraite, qui récapitule en les mettant côte à côte comme dans un portrait de famille, les personnages qui marquèrent durant quelque trois cent cinquante ans, l'histoire du pays.

Si le premier tableau se réfère à un texte précis, la dernière fresque contient, pour ainsi dire, tout un rayon de bibliothèque... L'instantané pictural concentre donc en lui une durée qui va de quelques heures à quelques siècles, ce qui témoigne avec éloquence de la puissance d'évocation de la représentation picturale. 
La formulation picturale de Charles Huot, dans ses tableaux d'histoire, doit donc se confronter avec le problème général de ce genre, à savoir rendre dans la statique d'un seul cliché, la dynamique de l'action représentée.

Dans la figuration de la scène du «débat sur la langue du 21 janvier 1793,» Huot a choisi de fixer le moment de la représentation durant le discours de Lotbinière contre la proposition d'amendement qui voulait l'anglais comme seule langue officielle et légale. L'action spécifique ici en est donc une de parole, ce que le tableau montre, c'est la durée d'un discours.

Pour ce faire, le peintre a recours au code stéréotypé des postures rhétoriques, selon lequel la levée du bras dans un geste déployé figure la déclamation; par ce biais, il traduit en termes spatiaux et visuels, ce qui se déroule dans le registre du langage, la toile ne se prêtant pas à la citation. L'action peinte suppose donc, par la médiation du titre ${ }^{1}$, une référence à la culture historique du spectateur qui, en voyant la scène, saura de quoi parle cet orateur debout dans le mutisme de sa représentation; il parle d'une langue qui est celle-là même dans laquelle il s'exprime. Il n'est pas facile à la peinture de prendre sa place dans ce sujet de part en part oral et lié au langage. De tels sujets sont donc particulièrement le lieu d'une réflexion sur la traduction picturale de significations verbales, puisqu'ils posent de façon aiguë la question: comment la peinture peut-elle tenir un discours?

La temporalité exacte choisie par Huot nous amène à repenser les modifications ou «erreurs historiques» que le peintre a introduites dans son tableau: les critiques contemporains d'Huot louaient la véracité de l'œuvre et sa grande fidélité à l'histoire: «Je ne crois pas me tromper, écrit Hormisdas Magnan, en disant que l'on cherchait en vain un anachronisme dans ce beau travail.» ${ }^{2}$ Robert Derome passe la reconstitution historique du «Débat sur les langues...» au crible de la critique, et découvre dans quelques accessoires du décor (fauteuil, chaises, costumes militaires) des «erreurs" quant à l'histoire (voir pages 25 et 26); de même, il note que par les

1 La fluctuation des titres de ce tableau est très significative: un titre moins explicite, mettant moins en évidence l'événement («Premier Parlement Canadien»... par exemple), était plus apte à faite taire «les mauvaises langues...».

2 Hormisdas Magnan, Charles Huot artiste peintre, sa vie, sa carrière, ses auvres, sa mort (Québec 1932), 31. 
fenêtres, Huot nous fait voir le Château Saint-Louis et la Citadelle, au lieu des jardins du Séminaire qui devraient y être; en 1793, la Citadelle, dont la première pierre n'est posée qu'en 1820, n'était certes pas dans le décor. Plus important quant à l'action dépeinte, Derome relève une autre liberté prise avec la réalité historique, celle du nombre de députés présents: «Huot a représenté quarante-neuf députés. ...Toutefois, seulement trente-neuf membres de l'Assemblée ont voté lors de la séance du 21 janvier 1793. Le peintre a donc choisi de faire une synthèse en incluant tous les députés élus plutôt que les seuls membres présents. $»^{3}$

Une fois déterminé avec précision le moment de la représentation, qui est celui du discours prononcé et non, par exemple, celui de la votation elle-même, cette modification mathématique s'atténue grandement, tous les élus ayant fort bien pu participer aux débats bien que trente-neuf seulement prirent effectivement part au vote qui rejeta l'amendement. C'est dans la durée condensée de l'action peinte que le passage de quarante-neuf élus représentés à trente-neuf votants, s'effectue, la tactique de fuir le vote étant, encore de nos jours, souvent utilisée.

Cette durée condensée dans la figuration, ce sont les textes des commentateurs qui la déploient et la spécifient en un avant, un pendant et un après événementiels. Voici par exemple la description que donne de ce tableau, Hormisdas Magnan:

Sur le trône présidentiel, est Jn-Antoine Panet, fidèle défenseur des prérogatives de sa nation. Debout, et dans un geste superbe, C. de Lotbinière revendique les droits du français. L'assemblée l'écoute avec une grande attention. Tous sont en proie à une vive émotion: les Canadiens sont debout ou assis, haletants d'émotion, sentant bien qu'ils sortiront victorieux de cette fameuse lutte oratoire. Les députés anglais, calmes en apparence, écoutent avec non moins d'attention et semblent prévoir l'issue de cette joute superbe qui sera le maintien officiel de la langue des premiers défricheurs du beau pays qu'ils viennent de conquérir. Dans le jubé, la foule semble écouter avec un grand intérêt. Une chaise, renversée sur le parquet de la Chambre indique bien la lutte, le mouvement et l'action, etc. ${ }^{4}$

Le texte, dans sa narration dramatique, joue avec la temporalité unique du tableau, reconstituant un passé, les discussions

3 Robert Derome, Bulletin de la Galerie Nationale du Canada (Ottawa 1976), no 2: 28 .

4 Hormisdas Magnan, op. cit., 29. 
déjà tenues auparavant dont la chaise renversée est la trace signifiante, et aussi un avenir, un «après» l'action dépeinte, soit la votation et le maintien du français par la défaite de l'amendement. Cette défaite à venir serait d'ailleurs déjà lisible chez les députés anglais, qui ne seraient que «calmes en apparence»: en effet, si l'apparence peut se voir, l'après-toile ne peut que se prévoir et être connu par la lecture des textes concernant l'événement peint. La dynamique de l'action, ses conséquences et ses effets, relèvent donc des textes et non de la représentation picturale figurée.

Il en va de même des effets que le tableau est dit provoquer sur les spectateurs qui le contemplent: la scène représentée, soit les effets de la rhétorique de Lotbinière, l'est dans un tableau qui veut lui aussi tenir aux yeux des spectateurs, un discours persuasif. Le tableau est, pour plusieurs critiques, une «leçon de courage et de patriotisme», mais il ne peut l'être que si quelqu'un le fait parler, et prononce la leçon qu'il contient.

Si l'idéologie représentée convient bien aux élites qui commanditèrent la toile historique de Huot, il faut noter que la conception de la peinture qui est la sienne, dans son éclectisme, date de quelque deux siècles; même si Huot a étudié à Paris de 1874 à 1886, l'enseignement reçu et les principes esthétiques transmis à l'École remontent aux formulations de l'Académie, à la fin du XVII $^{\mathrm{e}}$ siècle. «Le peintre, écrivait par exemple Roger de Piles, doit persuader les yeux comme un homme éloquent doit toucher le cœur. ${ }^{5}$ Le sujet rhétorique traité par Huot s'exprime dans une esthétique picturale qui elle-même se pense en termes de rhétorique et de persuasion, ce qui est souvent la conséquence d'un genre qui prend sa source dans le discours historique.

Sur la relation qui va de «peinture» à «histoire», nous lisons ce qui suit chez André Félibien (1619-1695) théoricien de l'Académie:

Le peintre n'est pas moins peintre pour ignorer l'histoire: aussi un peintre peut être fort habile dans son art et fort ignorant dans l'histoire. Que si je veux apprendre l'histoire, ce n'est point un peintre que je consulterai, il n'est historien que par accident; mais je lirai les livres qui en traitent expressément. ${ }^{6}$

5 Cité dans Bernard Teyssèdre, Roger de Piles et les débats du coloris au siècle de Louis XIV (la Bibliothèque des Arts, Paris, 1957), 258.

6 André Félibien, "L'idée du peintre parfait», in J. Charpier et P. Seghers, L'Art de la Peinture (Sehers, Paris 1957), 248-249. 
$L^{\prime}$ «essence» du travail pictural ne réside donc pas dans «la fidélité à l'histoire»; néanmoins, c'est à cause du public, de la clientèle, que le peintre doit se préoccuper de la vérité historique, s'il veut plaire à tout le monde :

Mais quoique le peintre représente la nature par essence et l'histoire par accident, cet accident ne lui doit pas être de moindre considération que l'essence, s'il veut plaire à tout le monde, et surtout aux gens de Lettres et à ceux qui considèrent un tableau plutôt par l'esprit que par les yeux et font principalement consister la perfection à représenter l'histoire et à exprimer les passions. ${ }^{7}$

La députation québécoise au début du $\mathrm{XX}^{\mathrm{e}}$ siècle s'apparentant bien sûr à la catégorie des "gens de lettres», c'est assurément au critère de l'histoire que les députés jugent la toile de Charles Huot. Les commentateurs traitent beaucoup plus longuement des événements historiques eux-mêmes que de leur figuration picturale, la principale qualité du tableau étant de faire voir le passé national. Un journaliste de La Presse précise que la peinture de Huot s'adresse à la fois «aux admirateurs d'art et à ceux qui aiment à voir évoquer notre histoire ${ }^{8}$. Parmi les membres du gouvernement qui commanditent l'œuvre, et dans le public en général, on peut croire vraisemblablement que le groupe des «amants de notre histoire» est plus nombreux que celui des «amateurs d'art».

Cette conception du tableau, témoin muet mais visible de l'histoire, qui, comme l'écrit avec envie Napoléon Bourassa, «retraçait sous le regard de tout un peuple, pour une suite de générations des gloires nationales, des vérités universelles » ${ }^{9}$, fait partie d'une théorie de la peinture qui dans la seconde moitié du $\mathrm{XIX}^{\mathrm{e}}$ siècle, est déjà fortement mise en brèche; pour un Huot, ces refusés ou indépendants peignent des œuvres où « règnent la pornographie et le bolchevisme» ${ }^{10}$. Aussi ne sommes-nous par surpris d'apprendre du biographe de Huot, Hormisdas Magnan, que «Huot avait le culte des maîtres de la peinture des siècles derniers. Il avait en horreur les évolutionnaires de toutes nuances en peinture. Les classiques lui suffisaient.» ${ }^{11}$ Son esthétique et sa pratique picturale incarnent bien ses convictions.

7 André Félibien, op. cit., 250.

8 La Presse, samedi 13 novembre 1920, 14

377.

9 Napoléon Bourassa, "Causerie artistique», in Revue Canadienne, 2 (1865):

10 Jean Chauvin, Ateliers (1928), 203.

11 Hormisdas Magnan, op. cit., 15. 
Il nous semble profitable, en plus d'analyser l'idéologie véhiculée par les thèmes, comme le fait Robert Derome, de tenir aussi compte de l'idéologie que la pratique picturale applique et exemplifie. Tout comme en littérature, les mécanismes de la narration (comment on raconte) influence la fiction (ce que l'on raconte), l'idéologie d'un tableau se situe à la fois dans sa facture (figuration) et dans son contenu (représentation).

Pour la clarté des analyses il nous semble qu'il y a avantage à distinguer la signification peinte de la signification textuelle où elle se source et qui s'y greffe par la prolifération du commentaire. En effet les commentateurs ont vite fait d'aller de la toile aux textes qui la sous-tendent, sans avertissement, convertissant sans heurt le tableau en «page d'histoire»; par exemple, Hormisdas Magnan écrira de la fresque de Huot «Je me souviens», «on peut lire, pour ainsi dire, écrite en langage imagé une série d'évocations historiques de deux grandes périodes de notre histoire nationale ${ }^{12}$, et cette même métaphore de l'écriture est omniprésente chez les commentateurs pour culminer dans cette affirmation de Maurice Hébert:

M. Charles Huot a voulu que ses peintures fussent des livres ouverts dans lesquels chacun put lire: nous avons lu et nous avons compris. ${ }^{13}$

La peinture comme instrument d'une pédagogie populaire, où le voir suffit et remplace le lire; ici encore cette conception datait: en 1815, Quatromère de Quincy la résumait ainsi:

Aux yeux du vrai philosophe, les arts sont les historiens populaires d'un grand nombre de faits, d'opinions et de traditions qui composent l'existence morale des nations... Ces livres originaux (les monuments de l'art), toujours ouverts à la curiosité publique, portent leur inspiration au dehors et la communiquent sans réserve au sentiment qui les consulte sans effort ${ }^{14}$.

La peinture d'histoire: un livre ouvert et exposé à toute la collectivité: c'est bel et bien en ces termes qu'est reçue par la critique la toile de Huot:

12 Hormisdas Magnan, op. cit., 31.

13 Maurice Hébert, «La peinture murale et la peinture du plafond de la salle de l'Assemblée législative», in Le Terroir, VI, 3 (juillet 1925). Cité par Hormisdas Magnan, op. cit., 32.

14 Quatromère de Quincy, Considérations morales sur la destination des ouvrages de l'Art (Paris 1815), 55. 
C'est un livre ouvert qui restera tout aussi bien pour perpétuer la mémoire de nos courageux ancêtres que pour rappeler les hommes d'État présents qui ont eu la bonne inspiration de l'y placer d'une façon si éloquente ${ }^{15}$;

par le biais de cette force persuasive, par le biais des «effets» sur les spectateurs, le passé représenté se trouve soudain réactivé et investi de significations qui se greffent au tableau à partir du présent de sa production ou de sa contemplation. Le 11 novembre 1913, on inaugure la toile de Huot, "Le débat sur les langues...», soit quelques années après la fondation du Devoir, et après le célèbre discours à Notre-Dame où le même Henri Bourassa défendit notre droit d'être catholique «en français». Posant la question «pourquoi cette toile satisfait-elle ceux qui l'ont commandée?», il est plus aisé de répondre par rapport aux thèmes dont elle traite (la représentation), que par rapport à l'esthétique (figuration) à laquelle elle appartient; à ce niveau, nous nous contenterons de noter:

1) qu'il a adéquation entre l'espace figural déployé et le lieu même d'exposition, la toile constituant en quelque sorte une «figure en abîme», soit l'assemblée dans l'assemblée, et la mémoire de la «salle des débats» se souvenant.

2) que le tableau inscrit dans sa facture les «représentants» de ses destinataires, les députés du présent contemplant les députés d'antan; si l'on tient compte des parentés qui relient les uns aux autres, le tableau est pour plusieurs élus du «portrait de famille», et joue la fonction d'une auto-représentation narcissique dans laquelle le «réalisme» et la ressemblance ne peuvent qu'augmenter l'effet de miroir.

3) que la figuration adopte ici le genre «journal des débats illustré», se voulant précisément la défense et l'illustration d'un débat particulier: il y a donc parallélisme entre la figuration réalisée et la consignation des débats parlementaires.

Il faut dire qu'il est dans la nature des artistes de juger en artiste, c'est-à-dire de faire prévaloir dans leurs jugements, comme dans leurs ouvrages, le mérite du savoir et de l'exécution. Au contraire, il est dans la nature du public ou des hommes étrangers à la pratique des arts, d'y considérer et d'y applaudir par dessus tout les qualités qui correspondent au sentiment. ${ }^{16}$

15 Hormisdas Magnan, «Le Premier Parlement Canadien», in La Vérité, samedi, 1 er novembre 1913, 114.

16 Quatromère de Quincy, op. cit., 36-37. 
Une production picturale qui présuppose, comme condition de possibilité, un accord avec le «sentiment national», doit pouvoir nous renseigner sur l'état de celui-ci.

Le travail précis et documenté de Robert Derome, attire l'attention sur des productions qui, trop longtemps, ont échappé à toute évaluation ou analyse bien qu'elles soient porteuses d'une part importante de notre culture, en incarnant dans un cas concret, la relation art et politique: parce que les tableaux d'histoire sont la visibilité du «sur-moi» collectif dans lesquels une société choisit de se réfléchir et de se reconnaître sous le mode de l'idéalisation, ils constituent à notre avis, un apport réel à l'histoire intellectuelle. 